\title{
Mogibacterium timidum
}

National Cancer Institute

\section{Source}

National Cancer Institute. Mogibacterium timidum. NCI Thesaurus. Code C86899.

A species of oblig ately anaerobic, Gram positive, rod shaped bacterium assigned to the phylum Firmicutes. This species is catalase negative, does not hydrolyze arginine, does not reduce nitrate and is assachrolytic. M. timidum is found in the oral cavity and may be related to periodontitis. 